\title{
Multicouches nanométriques pour l'optique $X$ : quelques exemples applicables aux lasers $X$
}

\author{
P. Boher et P. Houdy
}

Laboratoires d'Electronique Philips (LEP), 22 Avenue Descartes, BP.15, 94453 Limeil-Brévann Cedex, France

\section{Abstract}

Nanometric multilayers have long been recognized as powerful devices to make optics in the soft $X$-ray range. $X$-ray laser application leads certainly to the most drastic requirements in terms of reflectivity and stability under high flux. State of the art results obtained using ultrahigh vacuum if-sputtering technique are presented for various wavelength ranges. Above the silicon $\mathrm{L}-\alpha$ line $(127 \AA), \mathrm{Mo} / \mathrm{Si}$ system shows the higher reflectivity at normal incidence ( $R>50 \%$ ), in spite of the occurance of thin silicide layers at each interface. Unfortunately, its themmal stability is very limited, since complete interdiffusion occurs above $400^{\circ} \mathrm{C}$. Si $/ \mathrm{Si} N_{x}$ and $\mathrm{Si} / \mathrm{Si} O_{x}$ systems lave a much better thermal stability $\left(\simeq 800^{\circ} \mathrm{C}\right.$ ), and a high selectivity which make them suitable for $X$-ray diagnosis. In the wavelength range $44-127 \AA$, carbon based multilayers have the best potentialities. Nevertheless the reflectivity is limited by the interface roughness due to the metallic layer crystallisation. $W / C, C r / C$ and $M o / C$ are certainly the best systems in terms of reflectivity and thermal stability. At short wavelength $(<44 \AA)$, interdiffused systems such as $\mathrm{W} / \mathrm{Si}$ offer the possibility to reduced the layer thicknesses at very low value $(d<20 \AA$ ). Its thermal stability is nevertheless limited to about $300^{\circ} \mathrm{C}$.

\section{Introduction}

L'utilisation d'empilements multicouches à l'échelle nanométrique comme réflecteurs de rayons $X$ mous et mi-durs a connu un grand dévelopnement ces dernières années. Parmi les applications concernécs on peut citer la spectrométric de fluorescence X, les utilisations du rayonnement synchrotron ou lastronomic $X$. L'utilisation de ce type de miroirs pour réaliser unc cavité laser dans la gamme des rayons $X$ mous reste cependant très difficile malgré les nombreux efforts déployés à l'heure actuelle. Les contraintes imposćcs par cette application sont en effet extrêmement drastiques: grande réflectivité, incidence normale, stabilité sous haut Mux, sans parler des optiques semi-transparentes sur substrats tìs minces. Lat situation est toutcfois plus prometteuse dans le cas des optiques pour cliagnostics où les spécifications sont moins sévères.

Après un bref rappel du principe des opliques multicouches et de l'effet des principales imperfections sur les performances des miroirs, ect article donnera quelques exemples de réalisations efrectuécs au LEP dans une gamme assez large de longueurs d'onde. L'évolution thermicue des empilements sera en particulier précisće dans la perspective d'une utilisation de type laser $X$.

\section{Principe des réflecteurs de Bragg et effet des principales imperfections.}

Dans la gamme des rayons $X$ mous $(10<\lambda<400 \AA$ ), Ics indices de tous les matériaux sont très peu différents de ccux du vide, ce qui conduit à des coefficients réflecteurs extrêmement faibles en dehors des incidences très rasantes. Comme l'a montré E. Spiller dès 1972 (1), l'altcrnance périodique de deux matériaux, l'un le plus absorbant possible et l'autre le plus transparent possible, permet cependant d'alteindre des coefficients réflecteurs de plusicurs dizaines de pourcents en situation 
d'interférences constructives, c'est à dire quand la loi de Bragg $(2 \mathrm{~d} \sin \theta=\lambda$ ) est vérifiée. Les performances théoriques de ce type d'empilement à̀ une longueur d'onde donnée ne dépendent que du contraste d'indice cntre les deux matériaux par l'intermédiaire d'expressions analytiques très simples publićcs par A. Vinogradov en 1977 (2). De ce fait, en utilisant les sections efficaces de diffusion de tous les éléments, tabulées en fonction de la longucur d'onde (3), il cst facile de déterminer les couples de matériaux les plus efficaces à chaque longucur d'oncle (4). Les meilleures combinaisons associent génćralement un cspaccur clont unc des raics d'émission est proche de la longueur d'onde de travail et un ćlément métallique faisant office de réflecteur.

L'influence des imperfections structurales des empilements récls peut çtre simulée en utilisant la théorie des équations de Fresnel (5). L'épaisseur des couches doit être reproductible à mieux que $0.1 \%$ près pour ćviter une perte sensible de réflectivité (6). L'interdiffusion entre matériaux ne devient par contre pénalisante que quand elle est du même ordre de grandeur que l'épaisseur des couches ce qui n'est souvent le cas que pour les périodicités très faibles $(d<30 \AA)$. Un autre défaut très fréquent est la rugosité d'interface qui accroil la diffusion et limite très rapidement la réflectivité pour les courtes longucurs d'onde. La manière la plus simple de prendre en compte ce défaut dans le calcul de la réflectivité théorique est d'introduire un facteur de Debye-Waller $D(7)$ de la rorme:

$$
R=R_{0} \times D=R_{0} e^{-4 k^{2} \sigma^{2}}=R_{0} e^{-\left(\frac{2 \pi m \sigma}{d}\right)^{2}}
$$

ou $R$ et $R_{0}$ sont respectivement les réflectivités avec et sans rugosité d'interface, $k$ est le module du vecteur de propagation du champ ćlectrique, $\sigma$ la rugosité, $d$ la période de l'empilement et $m$ l'ordre de Bragg considćré.

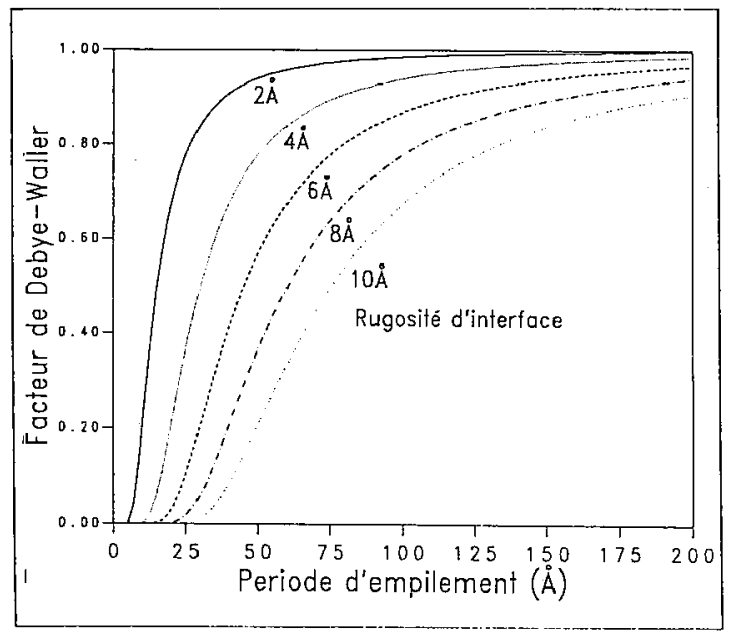

Les variations du facteur f:.D en fonction de la valeur de la période sont reportécs sur la figure I pour diffésentes valeurs de rugosité d'interfacc. La figure montre que l'influence de la rugositć d'ínterface est piépondérante dans le cas (l'empilements à coultes valeurs le periocle $(d<30 \AA)$ néccssaites à la réalisation d'empilement a incidence normale pour $\lambda<60$. Ellc est par contre bealcoup moins importante des que la période cst supéricur ì $60 \AA$.

Figure 1: Facteur de Debye-Waller pour diverses valeurs do rugosité d'interface en fonction de la période des empiliments.

\section{Méthodes expérimientales.}

Tous les systèmes multicouches décrits dans la suite ont été déposés par pulvérisation diode radiofréquence dans un bati de dépôt ultravide spécialement conçu pour disposer d'un contrôle liès précis des épaisscurs déposécs el des interfaces. Un 
dispositif original de pulvérisation pscudo-rćactive permet d'ćlargir les possibilités du système en autorisant le dépôt d'oxydes ou de nitrures à partir de cibles de métaux purs (8). Tous les paramètres de clépôt (pression totale, pression partielle tension d'autopolarisation des cibles ), sont régulés très précisément tout au long d'une expérience et les temps de dépôt sont controlés l'aide de volets rapides. Unc mesure en temps réel par ellipsométric cinćtique permet de suivre les processus de formation des interfaces (9), autorisant ainsi une optimisation facilc des conditions de croissance.

Les paramètres structuraux des empilements sont mesurés ex-situ après dépôt par réflectométrie de rayons $X$ rasants à la raic $K-\alpha$ du cuivre (10). La cristallinité est évaluće par le même appareillage cu configuration de cliffraction "stanclard", ainsi que par microscopic électronique à transmission haute résolution (11). Les performances optiques sont mesurées soil à l'aide d'un tube classique à la raic d'émission de divers éléments légers (12), soit à l'aide du rayonnement synchrotron (13). La stabilité thermique est ćvalućc par recuits successifs d'un même ćchantillon sous atmosphère neutre et mesurćc par réflectométric de rayons $X$ rasants.

\section{Résultats expérimentaux.}

\section{a) Grandes longueurs d'ondes $(\lambda>127 \AA)$.}

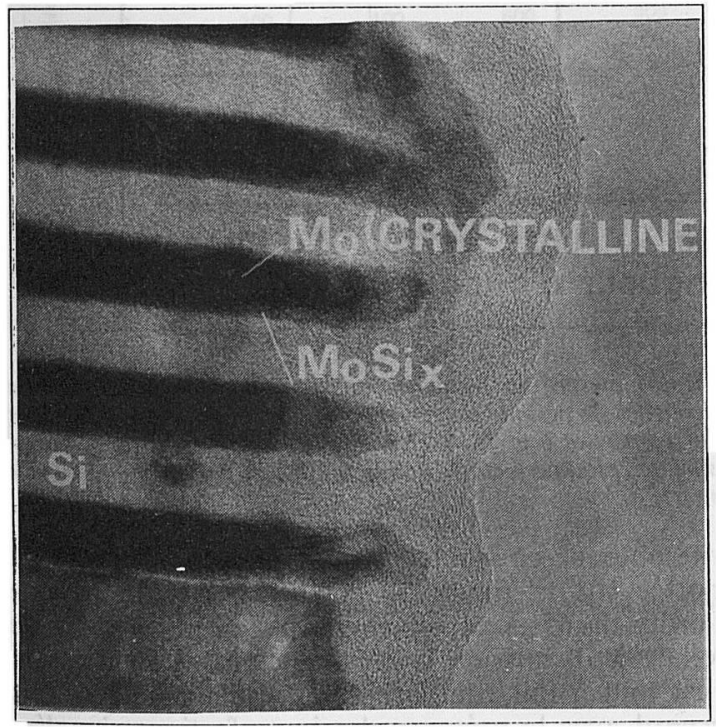

Figure 2: Cliché de microscoppie électronique di transmissiom

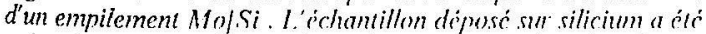
préparé par microclivage. la présenes de conches do siliciure amorphe à chaque interface est mise en évidenes ainri que litat cristallisé des couches de moljlidine.

Système Mo/Si.
Au dessus de la raie d'émission L- $\alpha$ du silicium, le couple de matćriaux domnant les plus fortes réflectivités, aussi bien thćoriques qu'expćrimentales, est Mo/Si. Le calcul de la réflectivité maximale en fonction de la longueur d'onde obtenue par la méthode de Vinogradoy (2), donnc en effet cles valeurs de réflectivité de l'ordre de $80 \%$ à la raie d'ćmission du silicium. La sćlectivité de ces empilements, optimisés en terme de reflectivité cst par contre relativement faible $(\lambda / \delta \lambda \simeq$ $20 \mathrm{cn}$ incidence normale). Dans la suite, nous décrivons des résultats expérimentaux obtenus alvec ce système ainsi qu'avec les systèmes $\mathrm{Si} / \mathrm{Si} N_{x}$ et $\mathrm{Si} / \mathrm{SiO}$, qui ont des sélectivités deux $\dot{a}$ trois fois plus importantes, et çui sont par consćcjuent potentiellement intercssants pour des applications de type diagnostic.

Après optimisation des conditions de dépôt, le système $\mathrm{Mo} / \mathrm{Si}$ présente toujours des couches interdiffusćes aux interfaces comme le montre la pholographic de microscopic à transmission de la figure 2. Dans ce cas. l'ćchantillon a élé microclivé, ce qui cxclu tout artefact de préparation. Les interfaces apparaissent avec une densité intermédiaire entre celle du molybdène ct celle du silicium. Il s'agit cn fait de couches 
de siliciure de molybdène dont la composition est voisine de $\mathrm{MoSi}_{2}$. Ces siliciures apparaissent spontanćment au cours de la croissance, comment nous l'avons démontré pour la prenic̀re fois par ellipsométric cinćtique in-situ (14). Les couches de siliciures sont asymétriques, la diffusion étant de l'ordre de $15 \AA$ à l'interface "Mo sur $\mathrm{Si}^{\prime \prime}$ et de l'ordre de $8 \AA$ à l'interface "Si sur Mo". Cette asymétrie constatée par d'autre auteurs (15), a été récemment simuléc en prenant en compte la cristallinité des couches (16).

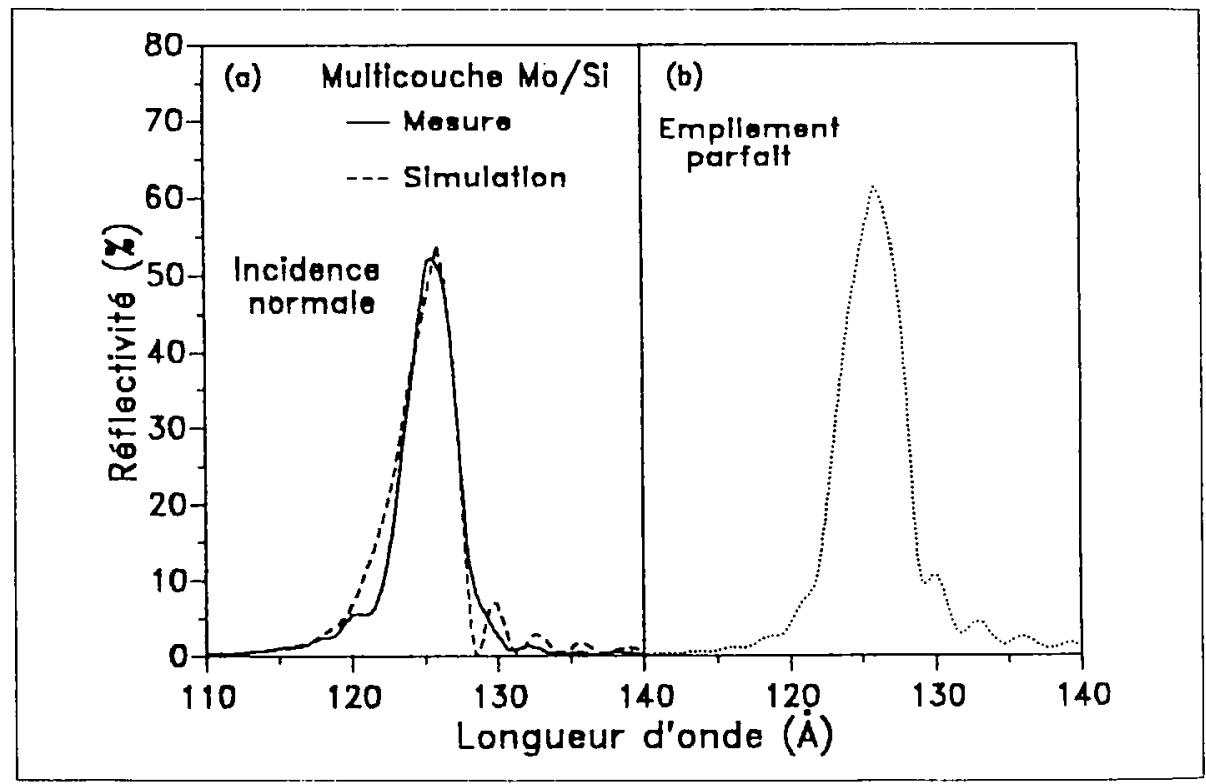

Figure 3: Réflectivité d'un cmpilenent Mo/Si mesurés en incidence quasi-nemrmale en fonction de la longueur d'onde. L'empiloment inclut 40 biconches do priviode mopenne $6.5 \AA$. L a simulation inchu deux couches d'interface asymétriques d'cipaisscur respertive 6 al $13 \AA$ el une dólive d'épaisseur de $I \AA$ d'um bout à l'autre de l'empilement. La sumpression de ces imperfections dome $\simeq 60^{\circ}$ '́ de réflectivité maximale (b) comparé aux $53 \%$ rócllement mesurés (a).

Un exemple de courbe cle réllectivité obicnuc en incidence quasi-normale à l'aide du rayonnement synchrotron est reportéc sur la figure 3. Dans ce cas, la période moyenne de f'empilement est de f'ordre de 6.5 \& ce qui donne un premier ordre de réflectivité de Bragg très proche de la raic d'emission L- $\alpha$ du silicium. Le maximum de réflectivité mesuré ( $\simeq 53 \%$ ) cst tout a fait comparable aux mcillcurs résultats publiés (17). Ce résultat est en accord avec la présence des imperfections signalées ci-dessus, auxquelles s'ajoutc une lérive d'épaisscur de l'ordre de $1 \AA$ de bas en haut de l'empilement, ct unc rugosité d'interface de $2.5 \AA$. La perte de réflectivité due à l'ensemble de ces imperfections est toutefois relativement limité (cf. figure 3), en accord avec les prévisions théoriçues précédentes (cf. figure 1).

\section{Systèmes $\mathrm{Si} / \mathrm{Si} N_{x}$ et $\mathrm{Si} / \mathrm{Si} O_{x}$.}

Dans le cas des systèmes $\mathrm{Si} / \mathrm{Si} N_{x}$ et $\mathrm{Si} / \mathrm{Si} O_{x}$ la grande quantitć de silicium présent dans les empilements permet d'obtenir unc absorption moyenne relativement faible au dessus du seuil L- $\alpha$ du silicium. Un grand nombre de couches participe à la réflectivité et la sćlectivité des miroirs est grandement amćlioréc (18). La réalisation pratique de ce type d'cmpilement par pulvérisation pseudo-réactive d'une seule cible 
de silicium permet d'obtenir des interfaces cxtrêmement planes ct une très bonne régularité des empilements. Les performances de ces miroirs dans la gamme des $\mathrm{X}$ mous sont tout à fait en accord avec les prévisions théoriques ( réflectivité supérieure à $20 \%$ en incidence normale ct sćlectivitć amćliorée d'un facteur deux à trois par rapport au systèmc classique $\mathrm{Mo} / \mathrm{Si}(18)$ )

Stabilité thermique comparée des systèmes $\mathrm{Mo} / \mathrm{Si}$ et $\mathrm{Si} / \mathrm{Si} N_{x}$.

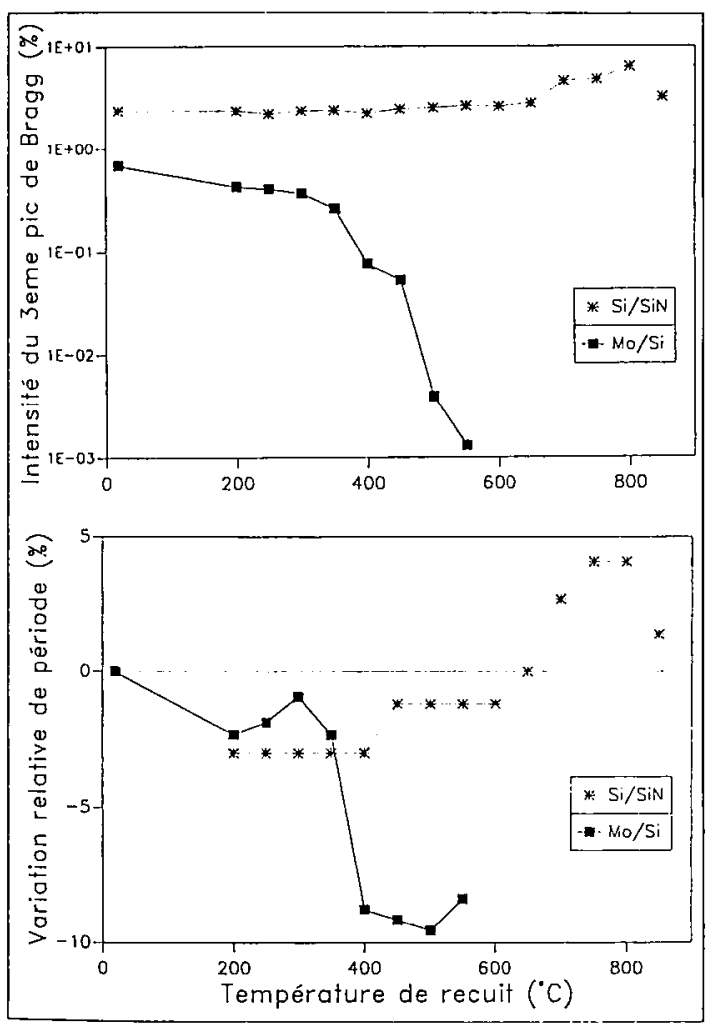

Figure 4: Evolution thermique des systèmes Mo/Si et SitSiN L'intensite du troisième pic de Bragg et la variation relatione de période évaluées par réflectométric de rayons $X$ rasants sont reportées en fonction de la tempćrature de iertit.
L'intérêt des empilements de $\mathrm{Si} / \mathrm{Si} N_{x}$ se situc aussi au niveau de Icur stabilité thermique exceptionnelle. En effet comme lc montre la figure 4 où ont été reportćes les évolutions thermiques comparées des deux systèmes, la structure multicouche se maintient après recuit à $800^{\circ} \mathrm{C}$. Dans le cas du système $\mathrm{Mo} / \mathrm{Si}$ au contraire la structure multicouche se dégrade progressivement dès recuit à $200^{\circ} \mathrm{C}$ et disparait complètement après recuit à $400^{\circ} \mathrm{C}$. Cette ćvolution est due à unc interdiffusion progressive des couches et à une cristallisation des couches de siliciure qui induit une contraction de période (cf. figure 4 ct ref (18)). L'évolution thermique des empilement $\mathrm{Si} / \mathrm{Si}$ $N_{x}$ est très différente car la qualité structurale semble $s^{\prime}$ amćliorcr après recuit à $800^{\circ} \mathrm{C}$ ct l'on constate unc dilatation de périnde. Des études sont en cours pour préciser ce mécanisme.

\section{b) Longueurs d'ondes moyennes $(44<\lambda<127 \AA)$.}

Dans cette gamme de longueurs d'oncle l'cspaccur le plus adapté cst le carbone du fait de son absorption relativement faible au dessus de son seuil d'émission $K-\alpha$. L'imperfection la plus pénalisante nour la réflectivité des miroirs est jei la rugosité d'interface qui doit être limitćc à moins de $3 \AA$ dans lc cas des miroirs à incidence normale. Le système lc plus étudić est assurément $W / C$ car il présente des interfaces relativement abruptes et unc faible rugositć (6). De plus les couches de carbone déposées par pulvérisation produiscnt un lissage efficace de la rugosité d'interface (19). L'alternance avec un mćtal de transition comme $\mathrm{Co}, \mathrm{Fc}, \mathrm{Cr}$ donne toutcfois les potentialités les plus importantes (4). D'autres systèmes tels $R h / C$ conduisent à des réflectivités théoriques élevécs jusqu'à $\lambda=100 \AA$. 
Cristallisation des couches métalliques.

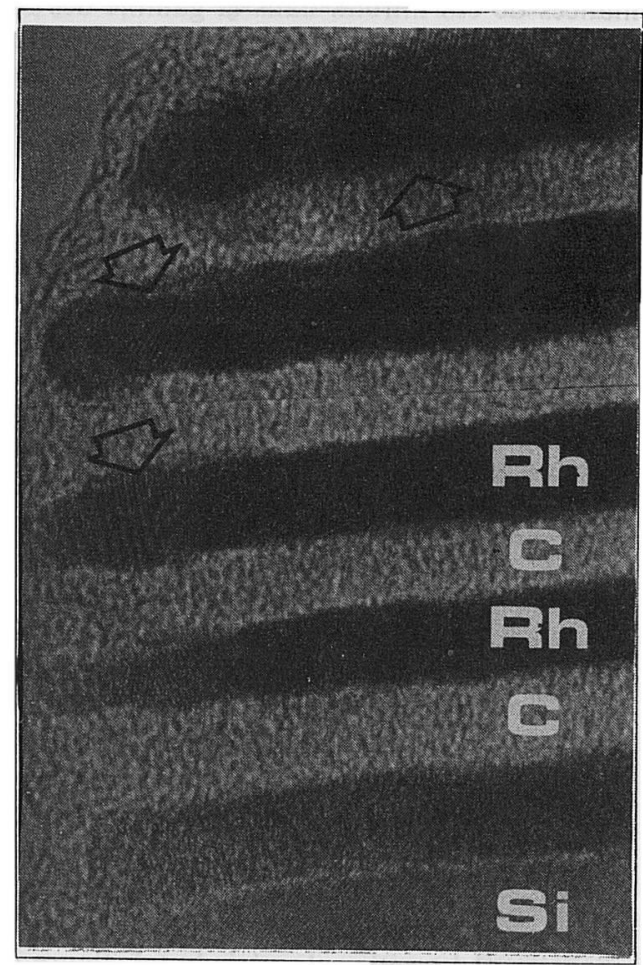

Figure 5: Cliché de microscopie électronique à tronsmission d'un empilement Rh/C. L'́rchantillon déposś sur silicium a été préparć par microclivege.
Dans bcaucoup de cas, les imperfections structurales résultent de la cristallisation des couches métalliques. Ceci induit une rugositć importante et limite dónc les performances, notamment lorsque les couches sont très minces. Cc phénomène est particulic̀rement important clans le cas du système $R h / C$ (20). L'observation par microscopic ćlectronique à transmission d'un ćchantillon de test comportant des couches de carbone d'épaisseur fixc ( $\simeq 50 \AA$ ), ct des couches de rhodium d'ćpaisscur variable ( dc 20 à $100 \AA$ ), est révélateur du phénomène de cristallisation (cf. figure 5). Les couches (le rhodium sont en effet l'autant plus cristallisces que leur ćpaisscur est grande, et l'on détecte même des cristaux de rhodium à l'intéricur des couches de carbone (llèches sur figure 5). Cet effet probablement dû à une trop grande quantité d'énergic présente à la surface peut être limité en adaptant les conditions de dépôt (21). II conditionne toutcfois l'ćvolution thermique de ces systèmes.

\section{Stabilité thermique comparćc de plusicurs systèmes à base de carbonc.}

L'évolution thermique des systèmes $H / C, M o / C, C H / C, F C / C, C o / C, N i / C$ et $R h / C$ est reportée sur la figure 6. Dans chacuc cas, nous avons reporté l'ćvolution de l'intensité du 3ème pie de Bragg et la variation relative de période mesurée en réflectométrie de rayons $X$ rasants en fonction de la température de recuit. La température de destruction a lacjuclle la réflectivité chute brutalement dépend de la nature de l'élément alterné avec le carbonc: clle varic de $300^{\circ} \mathrm{C}$ pour les éléments les plus sensibles à la cristallisation comme le rhodium $\mathrm{ct}$ le nickel, à plus de $600^{\circ} \mathrm{C}$ pour le tungstène. Toutes les évolutions thermiques à l'exception de celle $\mathrm{du} \mathrm{Cr} / \mathrm{C}$, s'accompagnent d'unc dilatation progressive des empilements. Cet effet déjà observé par plusicurs auteurs a ćtć attribuć à une dilatation des couches de carbone dûc à un changement de phase (22). Dans le cas du chrome il est probable que lá formation de carbure de chrome, relativement facile au vuc des donnécs thermodynamiques, induit une évolution opposćc. En tout état de cause, cette ćtude montre que le choix d'un miroir adapté pour unc application de type laser $X$ doit prendre en compte la stabilité thermique aussi hien que la qualité des interfaces réalisables. 


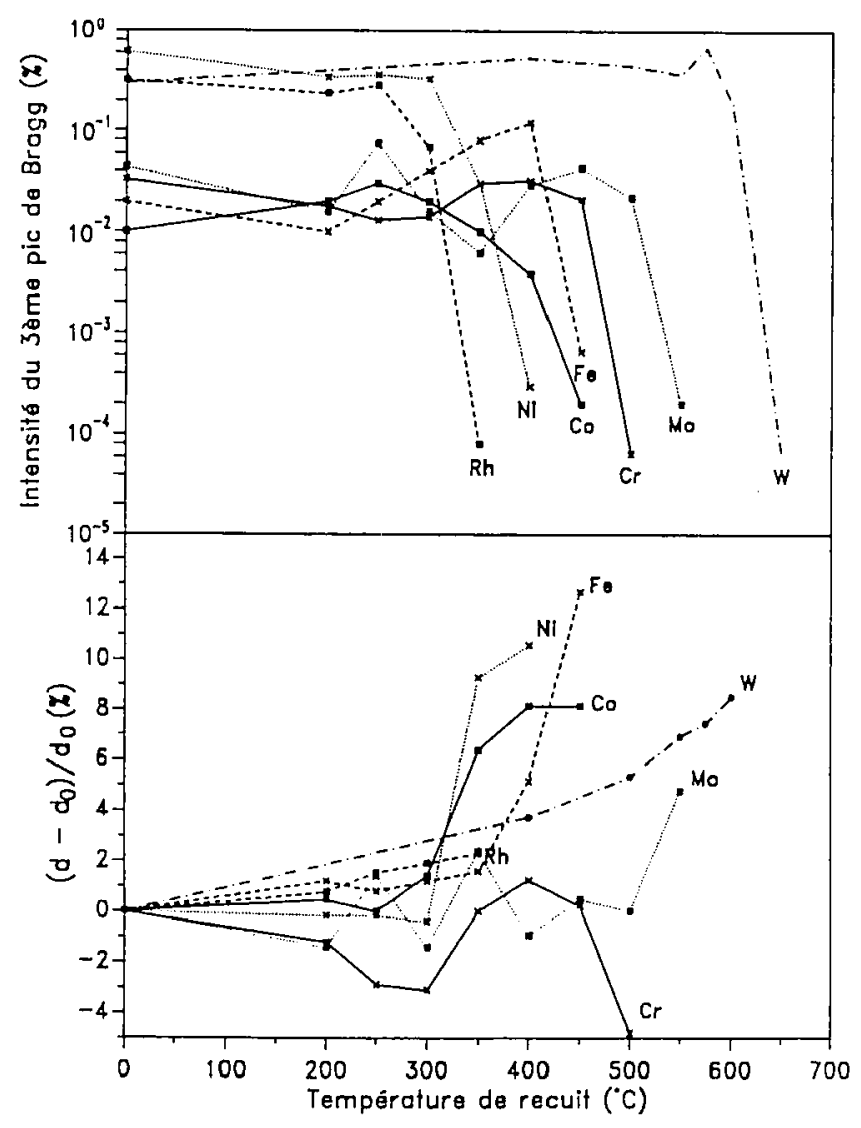

Fïgure 6: Evolulion thrmique de plusirurs sj'stèmes à base de carbone. L'intonsité du troisième pic de Bragg et la variation relative de période. civahérs par téllestométrie de rapons $X$ rasants, sont reportées en fonction de la température do recuit.

\section{c) Courtes longucurs d'onde $(\lambda<44 \AA)$.}

Dans ce cas, la réalisation d'optiques de grande quaiité sous incidence normale est pour l'instant hors de portéc car les épaisseurs de couches nécessaires deviennent extrèmement faible. Toutefois, le système $W^{\prime} / S i$ permet d'accéder à des périodes d'empilements relativement fajble sans augmentation trop important de la rugosité d'interface (23). Ceci est dû à unc forte interdiffusion entre le silicium ct le tungstène qui évite des phénomènes de rugosification comme le cristallisation ou la croissance en ilots. La contrepartic à cet effet est un contraste d'indice réduit entre couches du fait de la présence de silicium dans les couches de tungstène et donc des réflectivités notablement plus faibles que ne le prévoit le modèle sans imperfection.

\section{Evolution thermique du système $W / S i$.}

Par recuit in-situ sous vide, à l'jntéricur même d'un microscope à transmission à haute résolution, nous avons pu observer l'ćvolution structuralc d'un empilement 
W/Si de période très faible $(\mathrm{d} \simeq 25 \AA$ ). Comme le montre la figurc $7 . a$, l'échantillon après dépôt présente une bonne qualitć structurale avec unc rugosité d'interface relativement faible ( $\simeq 2.5 \AA$ par réflectomćtric de rayons $\mathrm{X}$ rasants $)$. L'ćpaisscur des couches de tungstène ( sombres ) apparaît plus grande que celle des couches de silicium (claires). Ceci est dû au processus d'interdiffusion car le rapport de composition calculé à partir des vitesses de dépôt est de l'ordre de 0.3 . Après recuit à $300^{\circ} \mathrm{C}$ (cf. figure 7.b), la structure multicouche est conscrvéc mais la rugositć d'interface a nettement augmenté. Cettc ćvolution s'accompagne d'une contraction de période constatée par réflectométric $X$, probablement due à un mécanisme d'interdiffusion et une cristallisation de la phase $W^{\prime} S i_{2}$ déjà constatéc par d'autres auteurs (23). Après recuit à $500^{\circ} \mathrm{C}$, la structure multicouche est complètement détruite (cf. figurc 7.c ).

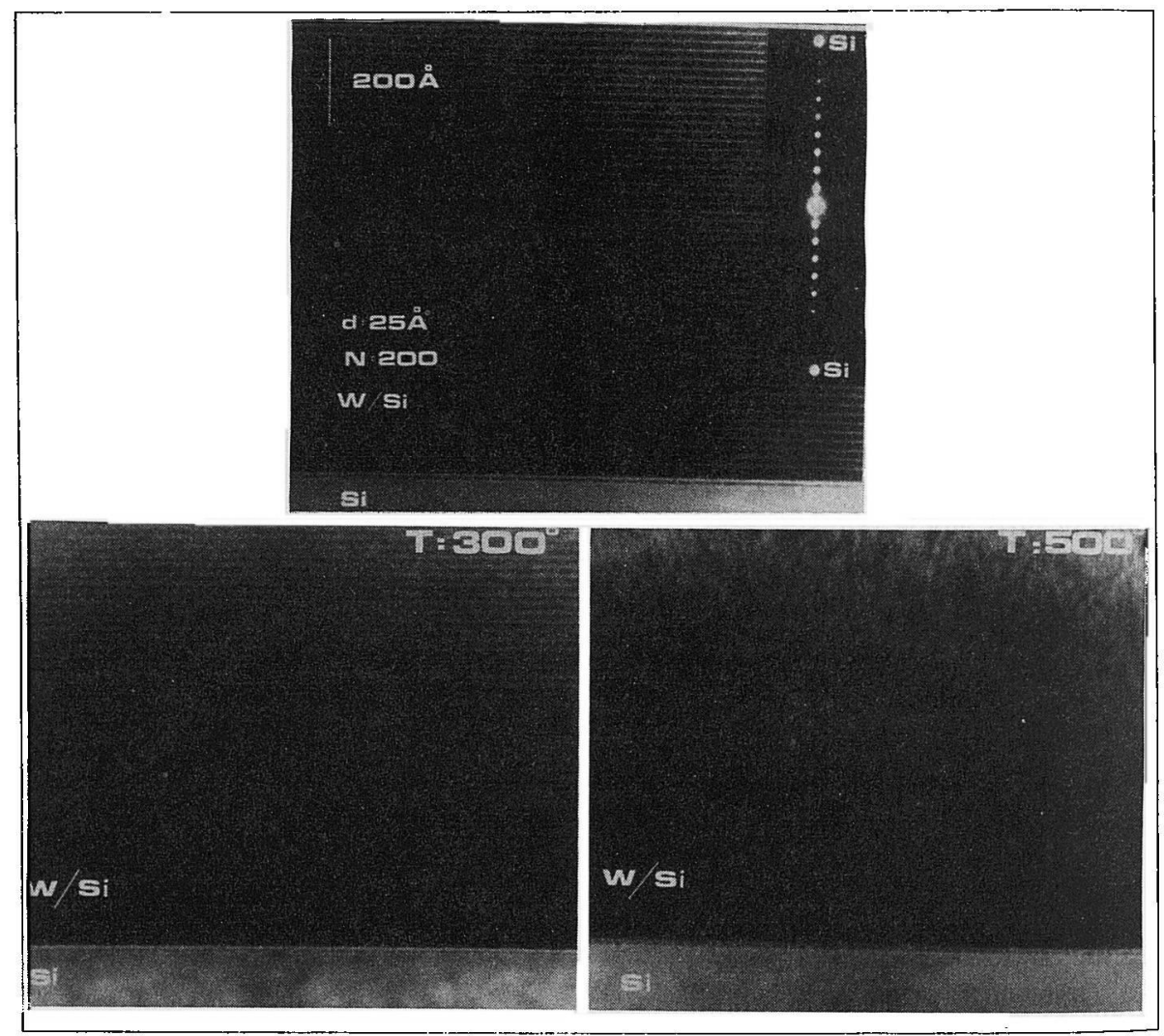

Figure 7: Evohtion structurale d'une multicourhe W'Si recuite in-situ dans un microscope à lransmission. Les étapes suivantes sont observées: (a) après dépìt, (b) après recuit à $300^{\circ} \mathrm{C}$, (c) après recuit à $500^{\circ} \mathrm{C}$. La période d'empilement est de lordre de $2.5 \AA$ et l'ompilement comporte 200 bicourhes.

\section{Conclusion.}

Comme l'ont montré les exemples ćvoqués dans cet article, la réalisation do multicouches nanométriques pour les optiques $X$ sc heurte à de nombreux problèmes: rugosité d'interface, couches d'interdiffusion, stabilité thermiçue limitéc... D'autre part, chaque longucur d'onde de travail nécessite l'ćtude de systèmes de matériaux différents, ce qui amplific généralement les problèmes. 
Pour les longueurs d'onde asscz grandes $(\lambda>127 \AA$ ), lc systc̀me de matériaux le plus efficace obtenu jusqu'ici cst $M o / S i$, qui permet, comme nous l'avons montré, d'obtenir des réflectivités maximales supéricures à $50 \%$. Ce système présente toutefois deux désavantages: sa stabilité thermique est très limitéc du fait de l'interdiffusion relativement facile entre les matériaux; ses performances deviennent relativement mauvaise pour des longucur's d'ondes supćricures à $250 \AA$ à cause de l'augmentation du coefficient d'absorption du silicium. L'utilisation de systèmes à base de nitrure comme $\mathrm{Si} / \mathrm{Si} N_{x}$ permet de régler en partic le premier problème en admettant toutefois une baisse de réflectivité. La résolution du sccond problème nécessitc l'utilisation d'un autre espaceur. Nous avons obtenu des résultats promettcurs avec le siliciure de magnésjum alterné soit avec du tungstènc, soit avec du nitrurc de silicium (24).

La gamme des longucurs d'onde moyennes ( $44 \AA<\lambda<127 \AA$ ) est bicn couverte par les empilements à base de carbonc. Nous avons montré que le choix de l'autre matériau devait prendre en comptc, à la fois la rugosité d'interface et la stabilité thermique. Dans ce contexte, les meilleurs systèmes semblent être des systèmes "classiques" comme $W / C$. ou $M o / C$. L'utilisation de carbure de bore à la place du carbone permet toutefois d'obtenir des résultats intéressants au dessus du scuil $\mathrm{K}-\alpha$ du bore $(67 \AA)(25)$.

Dans le domaine des courtes longueurs d'onde $(\lambda<44 \AA$ ) la possibilité de déposer des couches très minces sans augmentation notabic de rugosilć devient indispensable. Dans cette optique, les meilleurs systèmes sont $\mathrm{W} / \mathrm{Si}$ ou $\mathrm{W} / \mathrm{Mg}_{2} \mathrm{Si}(24)$ qui présentent une interdiffusion marquéc mais au prix d'unc baisse du contraste d'indice et donc des performances. La stabilitć thermique cst d'autre part limitćc toujours à cause des processus d'interdiffusion.

Les études futures semblent s'orienter suivant deux axes:

- Soit étudier des systèmes nouvcaux plus complexes à base de matériaux en phase amorphe (des carburcs par exemple).

- Soit s'intéresser à une approche "superéscaux" en essayant de réaliser des structures complètement épitaxiales.

qui devraient conduire à cles progrc̀s notables dans un futur prochc.

Les auteurs remercient les personnes suivantes qui ont participé activement à l'oblention des résultats: Mr. L. Hennet pour les études de stabilité thermique, Mr. P. Ruterana de l'Université de Caen pour les observations de microscopie électronique el Mr. M. Kôhne du synchrotron BlisSY à Berlin pour les mesures de réflectivilé.

\section{Références.}

1. "Multilayer interference coating for the racumm whraviolet", I.. Spiller, $\Lambda_{\Gamma}$ l. Phys. I ctt., 20, 365 (1972)

2. "X-ray and Far UV' mulilajer mimers: principler and possibilities". A.V. Vinogradov, B. Teldovich, Appl. Opt., 16, 89 (1977)

3. "Low energy X-ray interaction coefficients", B.I. IIcnke, Ntomic data and Nuclear data tables, 27, $\mathrm{N}^{\circ} 1(1982)$

4. "Reflecting properties of X-ray multilayer deviess", $\Lambda$. Rosenbluth, Thesis, Rochester University, Rochester N.Y. USA (1982)

5. "Recherches sur la propagation des ondes électromagnétiques sinusoidales dans les milieux stratifiés. Application aux couches minces", İ. Ahelés, Ann. de Physique, vol. S, 596 (1950)

6. "Performances of $C$ - $W$ multilavers as soft $X$-ray optics: high reflectivity in the range $2 d=60$ to 90 A ", Ph. Houdy, V. Bodart, C. Ilily, I'. Ruterana, I. Névot, M. Arbaoui, N. Alehyane, R. Barchewitz, SPIL $, 733,389$ (1986)

7. "Contribution à l'étude de cousches minces par réflexion spéculaire de raj!nns $X$ ", P. Croce, L. Névot, B. Pardo, Nouv. Rev. d'Optique Appliquéc, 3, 37 (1972) 
8. "Dinde rf sputtering of copper and copper oxide thin films and multilayers", P. Boher, Ph. I Ioudy, C. Schiller, L..J. Van Ijzendoorn, Thin Solid films, 174 (1989)

9. "Kinetic ellipsometry applied io soli X-ray mulilayer growth control". Ph. Iloudy, Revue de I'hysique $\Lambda$ ppliquce, vol. 23,1653 (1988)

10. "Grazing X-ray reflection analysis of namometric srale structure", P'. Boher, I'h. I Ioudy, C. Schiller, J. of $\Lambda$ ppl. Phys., 68, 61.33 (1990)

11. "Structure des interfaces, stude par microscopic à transmission ", l'. Rutcrana, 'Thèse de Doctorat, Université de Caen (1987)

12. "Versatile $X$-UV' spectrogiomeler with mulhilayer interference mirrors", M. Arbaoui, J.M. André, I'. Couillaud, R. Barchewit\%, Rev. Sci. Inst., 56, 2055 (1986)

13. J. Iïsher, M. Kühne, B. Wende, Nucl. Instr. Meth., $\Lambda 246,404$ (1986)

14. "Structural characteristics and perfimmances of if-sputtered $\mathrm{Mol} / \mathrm{Si}$ and $\mathrm{Co} / \mathrm{Si}$ multilayers for soft $X$-ray optics", P. Boher, I'h. IIoudy, I. I Iennct, M. Kühnc, P. Müller, C. Sctillou, J.C. Joud, I'. Ruterana, Proc. Sl'Il: 1547, 21 (1901)

15. "Magnetic, X-ray and TKM studics of screral multilay'er systems", M.B. Stearns, C.II. I se, C.II. Chang, Metallic multilayers and I pitaxy, lidited by M. Ilong, The Metallurgical Socicty, 55 (1988)

16. "Simulation of mixing at Mo/Si interfaces", J).B. Bocrcker, W.I. Morgan, Physics of X-ray multilayer structures, Jackson I lall, IVyoming, March 2-5 (1992)

17. "Molybdenum-silicon multiluper mirrors for the cxtreme ultraviolst". T.IV. Barbec, S. Mrowka, MC. Ilettrick, $\wedge$ ppl. Opt., 24, $883(1085)$

18. "Silicon / silicon nitride and silicon lsilicon oxide multilayers for X-UV' optical applications". P". Boher, Ph. I Ioudy, I. I Icnnct, J.P'. I) laboudinière, M. Külnne, P. Müller, ()ptical I'ngincering, 30 , $N^{\circ} 8,1049(1991)$

19. "Analysis of the smoothing process observed during the soft X-ray multilayer deposition", Ph. Iloudy, P. Boher, C. Ilily, R. Barchewit\%, N. Alchyanc, M. Ouahabi, Sl'll;, Vol.984, 95 (1988)

20. "Interface analysis of sputlered WJC, Rh/C and Ni/C multilayers for sof $X$-ray applications", P. Boher, Ith. Iloudy, C. Schiller, Thin Solid Films, 176, 161 (1989)

21. "Optimisation de multirouches numométriques: nomelles strutues el noweaux matérialex pour optiques à rayons $X$ mous", I.. I Icnnet, Ihc̀se de: Ioctorat, Université I'aris VI (1991)

22. "Expansion of amorphous cabon in WiC" multilapers after annealing". X..liang. D. Xian, Z. Wu, Appl. I'hys. I ctl., 57, 24, 2549 (1990)

23. "The structure of ulliathin C/W and Si/W multilayers for high performances in the soft X-ray optics ", P. Ruterina, J.P. Chevalicr, I'h. IIoudy, J. of Appl. Phys., 65, 3907 (1989)

24. "Characteristics and thermal behavior of $\mathrm{J} / \mathrm{Si}$ mililaycrs with well-defined interfaces", V. Dupuis, M.Г. Ravet, M. Piccuch, B. Vidal, J. of $\Lambda$ ppl. I'hys., 68, 3348 (199(I)

25. "Magnesium silicide based multilayers for soft X-rny" optics". P. Boher, Ph. IIoudy, L. Hcnnet, 7.G. Li, ^. Modak, D.J. Smith, M. Idir, 'T, Morcno, R. Barchewit\%. M. Kühnc, P. Müller, I.P. Delaboudiniète, Sl'II:, Vol. 1546, 5012 (1791)

26. "A comparative study of camben and horom cabide spacing lajers inside soft X-ray mirrors", $\mathrm{P}$. Boher, I'h. I Ioudy, I'. Kaïkati. R. Barchewilz. I .I. Van Jjendoom, 7.. (i. I.i, 1).J. Smith, J.C. Joud, SPII:, Vol. 1.34.3, $39(199(1)$ 